Rechtsmedizin 2022 $32: 391-395$

https://doi.org/10.1007/s00194-022-00558-0

Angenommen: 7. Januar 2022

Online publiziert: 8. Februar 2022

(c) Der/die Autor(en) 2022

\section{Isolierte bilaterale kranielle Hundebissverletzung bei einem Neugeborenen}

Elke Doberentz · Anja Wegner · Burkhard Madea

Institut für Rechtsmedizin, Universitätsklinikum Bonn, Bonn, Deutschland

\title{
Zusammenfassung
}

Durch Hundeangriffe können lebensgefährliche Verletzungen verursacht werden. Die Lokalisationen von Hundebissen korrelieren mit dem Alter und den motorischen Fähigkeiten des Opfers. Bei Kindern unter 6 Jahren finden sich im Vergleich zu Erwachsenen und älteren Kindern häufiger schwere Bisswunden, v. a. im Kopf-HalsBereich. Es wird der Fall eines 20 Tage alten Neugeborenen mit einer schweren Form der Osteogenesis imperfecta vorgestellt, das mit 2 Hunden allein gelassen und attackiert wurde und eine isolierte, bilaterale Kopfschwartenverletzung und intrakranielle Verletzungen erlitt. Besonderheiten von Hundebissen werden mit besonderem Augenmerk auf Säuglinge und Kinder diskutiert. Isolierte schwere Kopfverletzungen nach Hundebissen sind ein seltenes Phänomen.

\section{Schlüsselwörter}

Bisswunde $\cdot$ Hundeangriff $\cdot$ Kopfverletzung $\cdot$ Schädel-Hirn-Trauma $\cdot$ Osteogenesis imperfecta

Seit mehr als 15.000 Jahren leben Menschen mit Hunden zusammen [20]. Hunde sind die weltweit beliebtesten Haustiere, die als geliebte Familienmitglieder mit im Haushalt leben. Seit Domestizierung des Wolfs übernehmen Hunde mit ihren natürlichen und sozialen Fähigkeiten das Bewachen, Jagen und Hüten, in der gegenwärtigen Zeit auch das Aufspüren von Sprengstoff und Drogen, die Rettung vermisster Personen und die Unterstützung von Menschen mit Einschränkungen im Alltag [2, 6]. Hunde können ihre menschlichen Begleiter aber auch attackieren und lebensgefährliche Verletzungen verursachen [4, $5,7,17,22,23]$. In den Vereinigten Staaten von Amerika werden $80-90 \%$ aller Bissverletzungen von Hunden hervorgerufen [12]. In den meisten Fällen greifen Hunde ihre Besitzer oder eine dem Hund bekannte Person an [7, 8, 24]. Vor allem sehr junge Kinder sind aufgrund der Unerfahrenheit im Umgang mit Hunden besonders gefährdet, da sie nicht zwischen verspieltem, ängstlichem oder abwehrendem Verhalten des Hundes unterscheiden können $[3,19]$. Kinder können Hunde durch ungestüme, verspielte oder laute Verhal- tensweisen sogar provozieren und weisen längere Reaktionszeiten und eine geringere Abwehrfähigkeit auf [12]. Hundebisse betreffen in 60-70\% der Fälle deshalb Kinder; ein Viertel der Kinder ist hierbei jünger als 6 Jahre $[4,11]$.

\section{Falldarstellung}

\section{Vorgeschichte}

Ein 20 Tage altes männliches Neugeborenes schlief unbeaufsichtigt in Rückenlage in einer Schaukelwiege im Beisein von 2 Husky-Hündinnen. Der Kindsvater hörte es wimmern. Plötzlich vernahm er einen lauten Schrei und dann dauerhaftes Weinen. Er fand das Kind mit einer schweren Kopfverletzung in seiner Wiege vor und alarmierte den Rettungsdienst.

Nur einen Tag zuvor war das Neugeborene aus einem anderen Krankenhaus entlassen worden, wo es wegen Frakturen des linken Humerus und Femurs stationär aufgenommen worden war ( $\bullet$ Abb. 1a). Der Säugling hatte eine vorbekannte schwere, genetisch bestätigte Form der Osteogenesis imperfecta mit blauen Skleren, intra- 

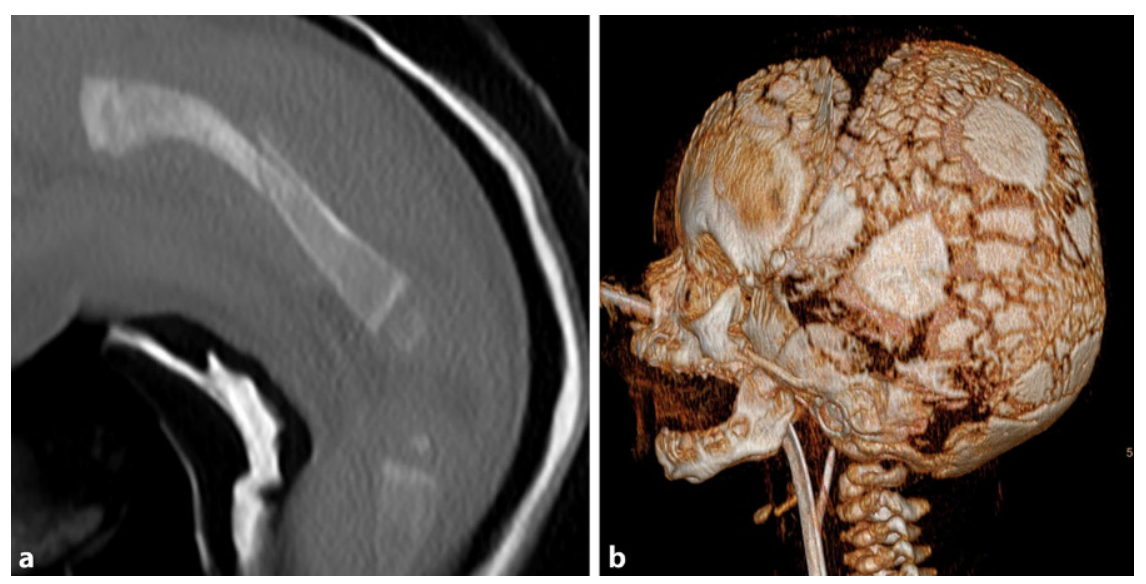

Abb. $1 \Delta$ a Computertomogramm des linken Beines mit frakturiertem linken Femur. Gekrümmte Röhrenknochen durch Osteogenesis imperfecta. b 3D-Computertomogramm des Schädels mit unterentwickeltem Schädeldach mit multiplen Ossifikationszentren

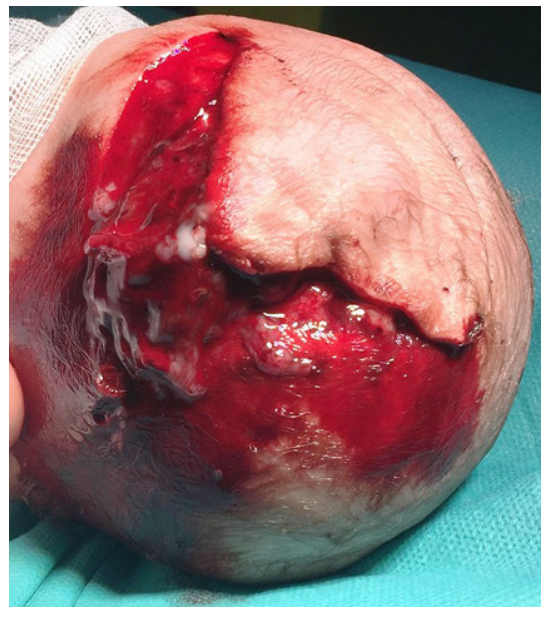

Abb. 3 ॥ Lange Kopfhautverletzung der linken Kopfseite mit freiliegendem Hirngewebe vor der Wundreinigung

uterinen Rippenbrüchen, kurzen gebogenen Röhrenknochen und unterentwickeltem Schädeldach (0 Abb. 1 b).

Zur Klärung der Verletzungsursache wurde eine rechtsmedizinische Untersuchung angeordnet. Die polizeiliche Tatortermittlung ergab, dass die Wiege eine Höhe von $90 \mathrm{~cm}$ aufwies. Die Bettwäsche wies Antragungen von Blut und Hirngewebe im Bereich der Lage des Kopfes des Neugeborenen auf. Beide Hündinnen hatten eine Schulterhöhe von ca. $55 \mathrm{~cm}$. Sie konnten ihre Schnauzen leicht in die Wiege legen, indem sie sich mit den Pfoten auf das Bettgitter auf einer Seite der Wiege stellten, wodurch die schwingende Wiege nach unten gedrückt wurde.

\section{Klinischer Befund}

Das Neugeborene wurde wach (GlasgowComa-Scale von 14 Punkten) in ein Krankenhaus eingeliefert. Es zeigte sich eine offene Schädel-Hirn-Verletzung mit bilateralen, horizontal und nach oberkopfwärts konkav verlaufenden, rissartigen Hautdurchtrennungen, links mit einer Länge von $13 \mathrm{~cm}$ und rechts von $4,5 \mathrm{~cm}$ (- Abb. 2, 3, 4 und 5). Die nach scheitelhöhenwärts weisenden Wundränder waren faltig aufgeschoben und aufklappbar. Die Dura mater wies vertikale rissartige Durchtrennungen auf, und Hirngewebe war freiliegend. Die Wundränder waren frei von Schürfungen oder Verletzungen der umgebenden Haut (• Abb. 6).

Das Kind wurde neurochirurgisch versorgt. Intraoperativ wurden Abstriche von den Wunden entnommen, wobei Streptococcus dysgalactiae, Schaalia (Actinomyces) canis und Neisseria animaloris nachgewiesen werden konnten. Eine augenärztliche Untersuchung ergab keine pathologischen Befunde. Bisher unentdeckte Frakturen wurden beim Skelettscreening nicht gefunden.

Zusätzlich wurden DNA-Proben aus der Mundschleimhaut beider Hunde entnommen. Bei einer Hündin konnte menschliche DNA nachgewiesen werden. Eine eindeutige Identifizierung des attackierenden Hundes war jedoch nicht möglich.

Das Neugeborene überlebte die schwere Kopfverletzung und konnte zu seiner Familie zurückkehren. Hinsichtlich der Hirnschädigung wurde eine gute Prognose ge-

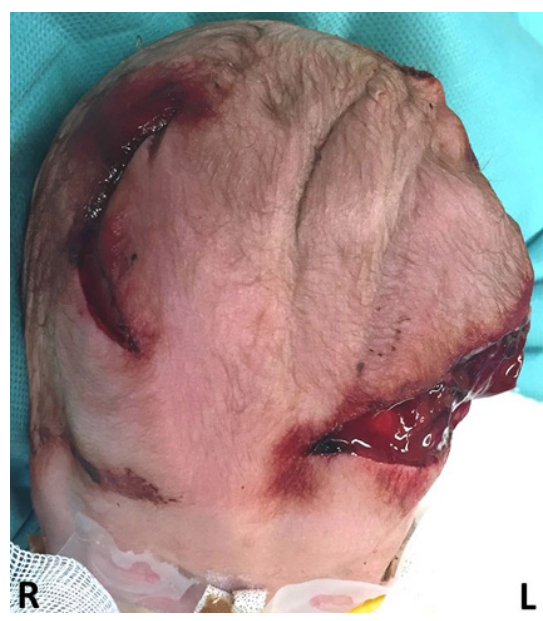

Abb. $2 \Delta$ Behaarte Kopfhaut mit 2 frontoparietalen Verletzungen ( $R$ rechts, $L$ links)

stellt. Eine der beiden Husky-Hündinnen wurde verdächtigt, das Kind attackiert zu haben, und fand in einer anderen Familie ein neues Zuhause.

\section{Diskussion}

Instinktives Schutz- und Territorialverhalten sowie besitz- oder angstinduzierte Aggression sind häufige Ursachen von Hundeangriffen [4]. Eine Vielzahl von Faktoren beeinflusst diese Instinkte, z. B. Alter und Geschlecht des Hundes, Gesundheitszustand, Sozialisation und Erfahrungen sowie Umgebung des Hundes, aber auch die Persönlichkeit des Hundebesitzers und die Interaktionen des Besitzers mit dem Hund [10]. Oft finden sich eine gestörte Beziehung zwischen Mensch und Tier und mangelnde Sozialisation des Hundes [15]. Bestimmte Hunderassen sind häufiger an schweren oder sogar tödlichen Hundeangriffen von Menschen beteiligt, wozu Deutsche Schäferhunde, Pitbulls und Rottweiler, aber auch Rassen, die als sozialisierter gelten, gehören, wie Labradore und Collies $[1,7,8,19,22] .75 \%$ aller Hundebisse werden von Rüden verursacht [15].

Die Lokalisation von Hundebissen korreliert mit der Körpergröße und den motorischen Fähigkeiten des Opfers [12, 15]. Im Gegensatz zu Erwachsenen weisen v. a. Kinder unter 6 Jahren schwere Bisswunden im Kopf-Hals-Bereich auf [7, 17, 23]. Hunde können aufgrund der kleineren Statur von Kindern den Kopf leicht erreichen, dementsprechend sind v. a. Nase und Lippen sowie Wangen und Ohren von Hundebissen be- 

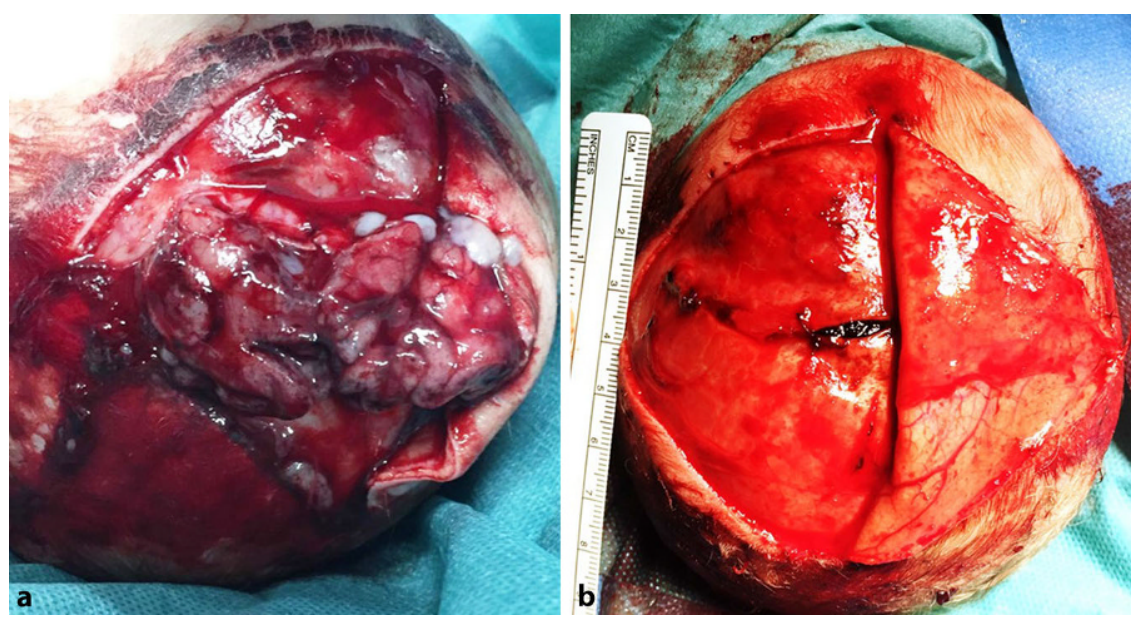

Abb. $4 \Delta$ Ansicht der Wunde der linken Kopfseite nach Aufklappen des oberen Wundrandes. a Freigelegtes Hirngewebe vor der Reinigung.b Blick auf den vertikalen Riss der Dura mater nach der Reinigung

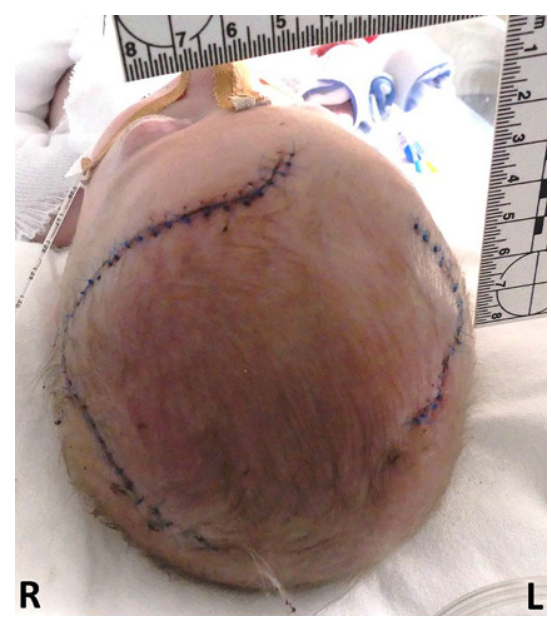

Abb. $6 \triangle$ Ansicht des Oberkopfes nach Reinigung und chirurgischer Behandlung ( $R$ rechts, L links)

troffen [23]. Ältere Kinder und Erwachsene präsentieren oft Bisse an den Extremitäten, wobei aufgrund von Abwehrverhalten, v. a. die Hände Verletzungen aufweisen [9]. Darüber hinaus können Verletzungen auftreten, wenn Hunde instinktiv versuchen, ihre Opfer zu Boden zu bringen [4].

Hundebisse verursachen Kompressionsverletzungen des Gewebes, mit oberflächlichen Schürf-, Schnitt- und Stichwunden, Prellungen, durch die Krallen verursachte Hautkratzer bis hin zu Gewebeausrissen mit Weichteilverlust, Verletzungen tieferer Strukturen und Knochenbrüchen $[3,4,7,8,13,17]$. Hunde neigen dazu, ihre Opfer zu zerren und zu schütteln, was die Gewebeschädigung durch auftretende Scherkräfte aggraviert $[5,7,15$,
24]. Penetrations- und Risswunden durch Eckzähne sind charakteristisch für Hundebisswunden, insbesondere, wenn sie von Gewebedefekten, Prellungen und linienförmigen Kratzspuren begleitet werden. Eine fehlende charakteristische Morphologie einer Hundebisswunde kann jedoch diagnostische Probleme verursachen. Im präsentierten Fall zeigte sich eine ungewöhnliche Morphologie, die zunächst an Schnittwunden erinnerte. Die Wunde wurde durch einen einzelnen Biss in den oberen Teil des Kopfes verursacht, der durch die Ober- und Unterkieferfangzähne jeweils einen Ausriss der Kopfschwarte und durch den Kieferschluss ein Aufschieben zur Scheitelhöhe hin mit vertikaler Verletzung der Dura mater verursachte. Ein Hundebiss kann einen Druck bis zu $14,06-28,12 \mathrm{~kg} / \mathrm{cm}^{2}$ verursachen und den dünnen Schädel eines Säuglings leicht perforieren $[9,13,23]$. Aufgrund der geringen Größe und Verformbarkeit des Schädels von Säuglingen und Kleinkindern können größere Hunde den gesamten Kopf in den Kiefer nehmen und schwere Verletzungen wie Gewebeausrisse, Perforationen und intrakranielle Verletzungen verursachen $[5,7,21]$. Schädelfrakturen finden sich meist bilateral und mit Hautrissen kombiniert [17, 21]. Das unterentwickelte Schädeldach erhöhte das Risiko intrakranieller Verletzungen. Derartige isolierte intrakranielle Hundebissverletzungen sind sehr selten $[17,18]$.

Die meisten Todesfälle durch Hundeattacken treten bei sehr kleinen Kindern mit

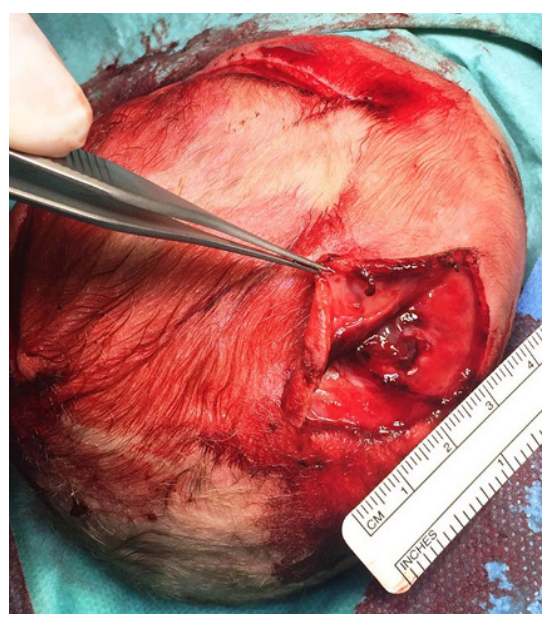

Abb. 5 \ Ansicht der Wunde der rechten Kopfseite nach Öffnen des oberen Wundrandes. Blick auf den vertikalen Riss der Dura mater

Hirn- und Gefäßverletzungen mit Blutverlust oder Luftembolie, Asphyxie und Halsmarkverletzungen auf $[4,5,7,22]$. Tsokos et al. berichten über den Fall eines 3 Wochen alten Säuglings, der in Gegenwart eines größeren Hundes auf einem Teppich schlief und enthauptet aufgefunden wurde [22]. Chu et al. berichten über 3 Fälle letaler Hundeangriffe [4]. Alle jungen Opfer wiesen typische Befunde schwerer Hundeangriffe mit mehreren Bissspuren und schweren Verletzungen der inneren Organe, darunter verschiedene Knochenbrüche auf. Ein $2 \frac{1}{2}$ Wochen altes Neugeborenes erlitt eine Schädelfraktur mit subgalealen und subarachnoidalen Blutungen. Ein weiteres $2 \frac{1}{2}$ Wochen altes Neugeborenes wies mehrere Punktionswunden am Kopf mit Schädelfraktur, subduraler und subarachnoidaler Blutung, kortikalen Kontusionen und intraventrikulären Blutungen auf. Diese Kinder waren, in Babywippen liegend, angegriffen worden. Diese Wippen waren mit einem elektrischen Mechanismus ausgestattet, sodass darin liegende Säuglinge ständiger Bewegung ausgesetzt sind, was einen Hund provozieren kann.

Im präsentierten Fall stellte sich die Frage, ob es sich um einen aggressiven Angriff gehandelt hat, zumal die Ankunft eines neuen Familienmitglieds Dominanzaggression auslösen kann, was einen Angriff bedingen kann, um die soziale Stellung des Hundes in der Familie zu behaupten [4]. Tödliche Hundeattacken sind durch heftige Angriffe mit wiederholtem Beißen und heftigem Schütteln gekenn- 
zeichnet [24]. Die Aggression des Hundes kann so heftig sein, dass es selbst mit zusätzlicher Hilfe anderer unmöglich ist, den Angriff zu stoppen [7, 24]. Im präsentierten Fall zeigte sich ein isolierter Biss in den Kopf des Neugeborenen. Auslöser für den Angriff war vermutlich das Wimmern des Säuglings, das das Interesse des Hundes weckte, in die Wiege zu schauen. Allerdings wurde die Wiege dann beim Aufrichten aktiv von den Pfoten des Hundes in Bewegung versetzt, und die daraus resultierende plötzliche Bewegung der Wiege könnte der Trigger für den Biss gewesen sein. Ein aggressiver Angriff hat somit vermutlich gar nicht vorgelegen.

Sind charakteristische Bissspuren vorhanden, kann ggf. eine Bissspurenanalyse den angreifenden Hund identifizieren [14]. Fotos der Wunden sollten mit einer rechtwinkligen ABFO-Skala (American Board of Forensic Odontology) angefertigt werden, um Verzerrungen durch nicht orthograde Aufnahmen zu kompensieren. DNA-Proben von den Bisswunden, der Kleidung des Opfers und dem Maul des verdächtigen Hundes können zur Identifizierung des Hundes beitragen [3]. Darüber hinaus kann in Bisswunden ggf. ein breites Spektrum aerober und anaerober Bakterien aus der Hundeschnauze nachgewiesen werden, die schwerwiegende Infektionen verursachen können [13, 24]. Intrakranielle Abszesse durch offene Kopfverletzungen nach einem Hundebiss wurden beschrieben [16].

\section{Fazit für die Praxis}

- Hunde sind sehr willkommene Familienmitglieder, aber der "beste Freund des Menschen" behält trotz Sozialisation seine Grundinstinkte und kann Menschen angreifen und lebensgefährliche Verletzungen verursachen.

- Häufigste Ursache von Hundeattacken sind gestörte Interaktionen zwischen Mensch und Hund.

- Vor allem Kinder sind gefährdet und erleiden häufig Bissverletzungen im KopfHals-Bereich.

- Hundebisswunden weisen meist Penetrationsverletzungen, Gewebequetschungen und Kratzspuren auf.

- Insbesondere Neugeborene und Säuglinge sollten niemals mit einem Hund unbeaufsichtigt bleiben.

\section{Isolated bilateral cranial dog bite injury in an infant}

Dogs can cause life-threatening injuries when they attack humans. The findings and observations used to identify dog bite wounds are presented. Compared with adults and older children, injured children under the age of 6 years are more likely to experience severe bite wounds in the head and neck regions. The case of a 20-dayold infant with a severe form of osteogenesis imperfecta, who was left alone with two dogs and was attacked is presented. The infant suffered a severe isolated head injury with bilateral scalp lacerations and intracranial injury. The peculiarities of dog bites are discussed with a special emphasis on infants and children. Isolated severe head injuries following dog bites are a rare phenomenon.

\section{Keywords}

Bite wound · Dog attack · Head injury · Craniocerebral trauma · Osteogenesis imperfecta

\section{Korrespondenzadresse}

\section{PD Dr. med. Elke Doberentz}

Institut für Rechtsmedizin, Universitätsklinikum Bonn

Stiftsplatz 12, 53111 Bonn, Deutschland

edoberentz@uni-bonn.de

Funding. Open Access funding enabled and organized by Projekt DEAL.

\section{Einhaltung ethischer Richtlinien}

Interessenkonflikt. E. Doberentz, A. Wegner und B. Madea geben an, dass kein Interessenkonflikt besteht.

Alle beschriebenen Untersuchungen am Menschen wurden mit Zustimmung der zuständigen EthikKommission, im Einklang mit nationalem Recht sowie gemäß der Deklaration von Helsinki von 1975 (in der aktuellen, überarbeiteten Fassung) durchgeführt. Für Bildmaterial oder anderweitige Angaben innerhalb des Manuskripts, über die Patienten zu identifizieren sind, liegt von ihnen und/oder ihren gesetzlichen Vertretern eine schriftliche Einwilligung vor.

Open Access. Dieser Artikel wird unter der Creative Commons Namensnennung 4.0 International Lizenz veröffentlicht, welche die Nutzung, Vervielfältigung, Bearbeitung, Verbreitung und Wiedergabe in jeglichem Medium und Format erlaubt, sofern Sie den/die ursprünglichen Autor(en) und die Quelle ordnungsgemäßnennen, einen Link zur Creative Commons Lizenz beifügen und angeben, ob Änderungen vorgenommen wurden.

Die in diesem Artikel enthaltenen Bilder und sonstiges Drittmaterial unterliegen ebenfalls der genannten Creative Commons Lizenz, sofern sich aus der Abbildungslegende nichts anderes ergibt. Sofern das betreffende Material nicht unter der genannten Creative Commons Lizenz steht und die betreffende Handlung nicht nach gesetzlichen Vorschriften erlaubt ist, ist für die oben aufgeführten Weiterverwendungen des Materials die Einwilligung des jeweiligen Rechteinhabers einzuholen.
Weitere Details zur Lizenz entnehmen Sie bitte der Lizenzinformation auf http://creativecommons.org/ licenses/by/4.0/deed.de.

\section{Literatur}

1. Bailey CM, Hinchcliff KM, Moore Z, Pu LLQ (2020) Dog bites in the United States from 1971 to 2018: a systematic review of the peer-reviewed literature. Plast Reconstr Surg 146:1166-1176

2. Bray EE, Otto CM, Udell MAR, Hall NJ, Johnston AM, MacLean EL (2021) Enhancing the selection and performance of working dogs. Front Vet Sci 8:644431

3. Byard RW (2016) Domestic dogs (canis lupus familiaris) and forensic practice. Forensic Sci Med Pathol 12:241-242

4. Chu AY, Ripple MG, Allan CH, Thogmartin JR, Fowler DR (2006) Fatal dog maulings associated with infant swings. J Forensic Sci 51:403-406

5. De Munnynck K, Van de Voorde W (2002) Forensic approach of fatal dog attacks: a case report and literature review. Int JLegal Med 116:295-300

6. Driscoll CA, Macdonald DW, O'Brien SJ (2009) From wild animals to domestic pets, an evolutionary view of domestication. Proc Natl Acad Sci USA 106:9971-9978

7. Heinze S, Feddersen-Petersen DK, Tsokos M, Buschmann C, Püschel K (2014) Fatal dog attacks on children. Actual genesis and motivation of fatal dog attacks demonstrated by specific casuistics and particular pathomorphological alterations. Rechtsmedizin 24:37-41

8. Horswell BB, Chahine CJ (2001) Dog bites of the face, head and neck in children. W V Med J 107:24-27

9. Kaye AE, Belz JM, Kirschner RE (2009) Pediatric dog bite injuries: a 5-year review of the experience at the Children's Hospital of Philadelphia. Plast Reconstr Surg 124:551-558

10. Kogan IR, Schoenfeld-Tacher RM, Hellyer PW, Oxley PA, Rishniw M (2019) Small animal veterinarians' perceptions, experiences, and views of common dog breeds, dog aggression, and breedspecific laws in the United States. Int J Environ Res Public Health 16:4081

11. Mathews JR, Lattal KA (1994) A behavioral analysis of dog bites to children. J Dev Behav Pediatr 15:44-52

12. Nygaard M, Dahlin LB (2011) Dog bite injuries to the hand.J Plast Surg Hand Surg 45:96-101 
13. O'Brien DC, Andre TB, Robinson AD, Squires LD, Tollefson TT (2015) Dog bites of the head and neck: an evaluation of a common pediatric trauma and associated treatment. Am J Otolaryngol 36(1):32-38

14. Pomara C, D'ErricoS, JarussiV, TurillazziE, FineschiV (2011) Cave canem: bite mark analysis in a fatal dog pack attack. Am J Forensic Med Pathol 32:50-54

15. Rothe K, Tsokos M, Handrick W (2015) Anima and human bite wounds. Dtsch Arztebl Int 112:433-443

16. Ruszin T, Vaszilkó M, Rásonyi-Kovács O, Ujpál M (2009) A rare case of fatal outcome due to extensive facial trauma caused by dogbite. Fogorv Sz 102:187-190

17. Saadi R, Oberman BS, Lighthall JG (2018) Dogbite-related craniofacial fractures among pediatric patients: a case series and review of literature. Craniomaxillofac Trauma Reconstr 11:249-255

18. Santana-Montero BL, Ahumada-Mendoza H, VacaRuíz MA, Castro-Sierra E, Sánchez-Herrera F, Fernández-Portilla E et al (2009) Cerebellar abscesses caused by dog bite: a case report. Childs NervSyst 25:1137-1141

19. Sarenbo S, Svensson PA (2021) Bitten or struck by dog: a rising number of fatalities in Europe, 1995-2016. Forensic Sci Int 318:110592

20. Shannon LM, Boyko RH, Castelhano M, Castelhano M, Corey E, Hayward JJ, McLean C, White ME, Said AM, Anita BA et al (2015) Genetic structure in village dogs reveals a Central Asian domestication origin. Proc Natl Acad Sci USA 112:13639-13644

21. Steen T, Ravin K, Timmons S, Kershenovich A (2015) Intracranial injuries from dog bites in children. Pediatr Neurosurg 50:187-195

22. Tsokos M, Byard RW, Püschel K (2007) Extensive and mutilating craniofacial trauma involving defleshing and decapitation: unusual features of fatal dog attacks in the young. Am J Forensic Med Pathol 28:131-136

23. Wei LA, Chen HH, Hink EM, Durairaj VD (2013) Pediatric facial fractures from dog bites. Adv Ophthalmic Plast Reconstr Surg 29:179-182

24. Wright JC (1985) Severe attacks by dogs: characteristics of the dogs, the victims, and the attack settings. Public Health Rep 100:55-61

Häßler F, Nedopil N, Dudeck M (Hrsg)

\section{Praxishandbuch Forensische Psychiatrie}

Berlin: Medizinisch Wissenschaftliche Verlagsgesellschaft 2022, 3., aktualisierte und erweiterte Auflage, 847 S., (ISBN: 978-3-95466-643-0), 199,95 EUR

\section{F. Häßler|N. Nedopil \\ M.Dudeck (Hrsg.)}

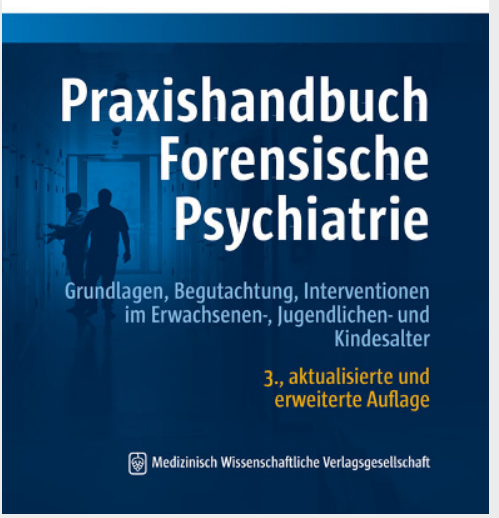

Das Praxishandbuch Forensische Psychiatrie, das deutschsprachige Standardwerk über die forensisch-psychiatrische Begutachtung und Intervention im Erwachsenen-, Jugendlichen- und Kindesalter, liegt nun in der 3. aktualisierten und erweiterten Auflage vor. Gegenüber der 2. Auflage von 2014 wurde das Buch um zahlreiche neue Themen wie die Forensische Psychiatrie im internationalen Vergleich, die Grundlagen der Verkehrsmedizin, rechtsmedizinische und rechtspsychologische Erörterungen, Testierfähigkeit oder neurobiologische Aspekte ergänzt und erweitert. Neben den drei Herausgebern haben sich 61 weitere Autorinnen und Autoren beteiligt. Das Buch ist grob in 2 Teile (Basiswissen, Praxiswissen) aufgeteilt, die wiederum in einzelne, klar umrissene Kapitel gegliedert sind. Ausführlich werden die Grundlagen der Begutachtung samt Qualifikation, Stellung des Gutachters im Prozess, Aufbau des Gutachtens, rechtlichen Grundlagen und ethischen Aspekten dargestellt. Die Praxis der Begutachtung nimmt den größten Teil ein. Sie wird zu einem deliktsbezogen, zum anderen nach den verschiedenen Störungen und ihrer möglichen forensischen Relevanz dargestellt. Einzelne Kapitel beschäftigen sich darüber hinaus mit den Ursachenkomplexen von Delinquenz oder der kriminalprognostischen Bewertung.
Verwandten Fächern wie der Rechtspsychologie und der Rechtsmedizin werden eigene Kapitel gewidmet; wobei von rechtsmedizinischer Seite die Themen Schuldfähigkeit bei Alkohol-, Drogen- und Substanzmissbrauch (Autor: Ulrich Hammer) und Verkehrsmedizin (Autoren: Gerd Laux, Alexander Brunnauer) behandelt werden.

Die Formen der Sicherung, die Behandlung von Straftätern, sozialrechtliche und zivilrechtliche Fragestellungen sind weitere Themen, denen eigenständige Kapitel gewidmet sind. Das Buch ist klar strukturiert, als Nachschlagewerk wie als Lehrbuch gleichermaßen geeignet und kann uneingeschränkt allen empfohlen werden, die sich mit forensisch-psychiatrischen Fragen beschäftigen.

M. Bohnert, Würzburg 